\title{
Fixed points of set-valued maps in locally complete spaces
}

\author{
Carlos Bosch ${ }^{1 *}$, César L García', Thomas Gilsdorf², Claudia Gómez-Wulschner ${ }^{1}$ and Rigoberto Vera
}

"Correspondence: bosch@itam.mx 'Departamento de Matemáticas, ITAM, Río Hondo 1, Ciudad de México, 01080, México

Full list of author information is available at the end of the article

\begin{abstract}
We prove an extension of the Pareto optimization criterion to locally complete locally convex vector spaces to guarantee the existence of fixed points of set-valued maps.
\end{abstract}

MSC: Primary 47H10; 47H04; secondary 46N10; 46A03

Keywords: fixed points; set-valued map; Pareto optimization

\section{Introduction}

Ekeland's variational principle, a milestone in the theory of nonlinear optimization, focuses on solving an optimization problem via a perturbed optimization problem. Since its appearance many extensions and equivalent formulations have been shown. Some of them, related to our discussion below, are contained in [1-8]. In [9] Azé and Corvellec generalized, in the setting of metric spaces, a result due to Lim ([10]) on the existence of fixed points for weakly inward multivalued contractions, defined on a nonempty closed subset of a Banach space. Their argument is remarkably simple: on the one hand, it avoids the use of transfinite induction (as in Lim's paper) and, on the other hand, it uses Ekeland's variational principle as a main tool to guarantee the existence of fixed points. Ekeland's principle has been shown equivalent to other optimization statements, in particular, and of interest in this paper, is the equivalence to an optimization criterion of Pareto (see [11]) for which the existence of optima (critical points for dynamical systems) has been shown in complete metric spaces (see [12]). Our main result (Theorem 3.2) is a modification of that optimization criterion of Pareto, which shows how the existence of fixed points for set-valued maps can be extended to the setting of locally complete spaces. Among the consequences of Theorem 3.2, we provide a simple argument of Azé and Corvellec's theorem ([9], Theorem 2.3). Although our results are set in the context of locally complete spaces, let us say that related to Ekeland's type variational principles, some other forms of completeness have been use in the literature (e.g., quasi-metric spaces with $Q$-functions, fuzzy metric spaces, or sequentially lower complete spaces). Some of these include equivalences to Ekeland's variational principle, for instance, to Caristi fixed point theorem or Takahashi minimization theorem, all of them with their natural connection to solution to equilibrium or fixed point theorems for set-valued maps (see, for instance [11, 13-19]).

(c) The Author(s) 2017. This article is distributed under the terms of the Creative Commons Attribution 4.0 International License (http://creativecommons.org/licenses/by/4.0/), which permits unrestricted use, distribution, and reproduction in any medium, provided you give appropriate credit to the original author(s) and the source, provide a link to the Creative Commons license, and indicate if changes were made. 


\section{Preliminaries}

Throughout this paper $(X, \tau)$ will denote a Hausdorff locally convex space where the topology $\tau$ is generated by a saturated family of seminorms $\left\{\rho_{j}\right\}_{j \in J}$. If $B$ is a subset of $X$ which is balanced and convex, we will call $B$ a disk. Let $X_{B}$ be the linear span of $B$, endowed with the topology generated by the Minkowski gauge of $B, \rho_{B}$. When $B$ is bounded $\rho_{B}$ is a norm, and the norm topology is finer than the topology inherited from $X$. If $\left(X_{B}, \rho_{B}\right)$ is a Banach space we say that $B$ is a Banach disk. We say that $X$ is a locally complete space if each closed, bounded disk is a Banach disk. Local complete spaces are also known as $c^{\infty}$ or convenient spaces. The notion of local completeness has become important in all sorts of applications, for instance, in nonlinear distribution theory (see $[1,20]$ and the references therein), or as a context where existence and uniqueness for nonlinear integro-differential equations can be shown (see [21]). By lsc we refer to a lower semicontinuous functions, $f: X \rightarrow \mathbb{R} \cup\{\infty\}$, which are proper, that is, their effective domain, $\operatorname{dom}(f):=\{x: f(x)<\infty\}$, is nonempty.

For a closed subset $A \subset X$ we will consider set-valued maps $T: A \rightarrow 2^{A}$. Such maps $T$ are known in the literature as dynamical systems and, in our discussion, points of interest are $x^{*} \in A$ such that $x^{*} \in T x^{*}$ (known as fixed points of $T$ ) and, especially, critical points of $T$ (points $x^{*} \in A$ such that $T x^{*}=\left\{x^{*}\right\}$ ). Let us start with the definition and properties of some functions that will be used in the sequel. For each $j \in J, \phi_{1 j}, \phi_{2 j}: X \rightarrow[0, \infty)$ will be the functions defined by

$$
\phi_{1 j}(x)=\rho_{j}-\operatorname{diam}(T x)=\sup \left\{\rho_{j}\left(y-y^{\prime}\right): y, y^{\prime} \in T x\right\}
$$

and

$$
\phi_{2 j}(x)=\rho_{j}-\operatorname{dist}(x, T x)=\inf \left\{\rho_{j}(x-y): y \in T x\right\} .
$$

Also, for each $j \in J$, and for every pair of subsets $A, B \subset X$, we define

$$
e_{j}(A, B)=\sup \left\{\rho_{j}(x-B): x \in A\right\}
$$

where $\rho_{j}(x-B)=\inf \left\{\rho_{j}(x-b): b \in B\right\}$, is the $\rho_{j}$-distance from $x$ to $B$. In general, we define

$$
\rho_{j}(A-B)=\inf \left\{\rho_{j}(a-B): a \in A\right\}=\inf \left\{\rho_{j}(b-A): b \in B\right\}=\rho_{j}(B-A),
$$

where $\rho_{j}(a-B)$ denotes the $\inf \left\{\rho_{j}(a-b): b \in B\right\}$ and $\rho_{j}(b-A)=\inf \left\{\rho_{j}(b-a): a \in A\right\}$.

It is important to note that, in general, $e_{j}(T x, T z) \neq e_{j}(T z, T x)$. For instance, take $X=\mathbb{R}^{2}$ equipped with the usual Euclidean norm $d$ and consider the subsets $A=\{(a, 2):-1 \leq a \leq$ $1\}$ and $B=\left\{(x, y): x^{2}+y^{2} \leq 1\right\}$. An easy computation shows that $e_{d}(A, B)=\sqrt{5}-1$ while $e_{d}(B, A)=3$.

Now we provide sufficient conditions on the functions $e_{j}$ to get lower semicontinuity and continuity properties for the functions $\phi_{1 j}$ and $\phi_{2 j}$. We start with the following.

Proposition 2.1 If $M_{j} \in \mathbb{R}^{+}$is such that $e_{j}(T x, T z) \leq M_{j} \rho_{j}(x-z)$ for every $x, z \in X$ then $\phi_{1 j}(x) \leq \phi_{1 j}(z)+2 M_{j} \rho_{j}(x-z)$. Hence, $\phi_{1 j}$ is $\rho_{j}$-continuous. In particular since $\tau$ is a uniformity we see that $\phi_{1 j}$ is $\tau$-uniformly continuous. 
Proof Let $x, z \in X$ and $y, y^{\prime} \in T x$. Then, for each $n \in \mathbb{N}$ choose $w_{n} \in T z$ such that

$$
\rho_{j}(y-T z)<\rho_{j}\left(y-w_{n}\right)<\rho_{j}(y-T z)+\frac{1}{n},
$$

and similarly for $y^{\prime}$ choose the corresponding $w_{n}^{\prime} \in T z$. Then

$$
\begin{aligned}
\rho_{j}\left(y-y^{\prime}\right) & \leq \rho_{j}\left(y-w_{n}\right)+\rho_{j}\left(w_{n}-w_{n}^{\prime}\right)+\rho_{j}\left(w_{n}^{\prime}-y^{\prime}\right) \\
& <\rho_{j}(y-T z)+\rho_{j}\left(w_{n}-w_{n}^{\prime}\right)+\rho_{j}\left(y^{\prime}-T z\right)+\frac{2}{n} \\
& \leq \phi_{1 j}(z)+2 e_{j}(T x, T z)+\frac{2}{n} .
\end{aligned}
$$

Thus, $\rho_{j}\left(y-y^{\prime}\right) \leq \phi_{1 j}(z)+2 e_{j}(T x, T z)$. By taking the supremum over all $y, y^{\prime}$, we obtain

$$
\phi_{1 j}(x) \leq \phi_{1 j}(z)+2 e_{j}(T x, T z) \leq \phi_{1 j}(z)+2 M_{j} \rho_{j}(x-z) .
$$

A similar argument shows that

$$
\phi_{1 j}(z)-\phi_{1 j}(x) \leq 2 M_{j} \rho_{j}(z-x)
$$

also holds. Hence $\phi_{1 j}$ is $\rho_{j}$-uniformly continuous.

For the function $\phi_{2 j}$, the result corresponding to Proposition 2.1 is as follows.

Proposition 2.2 If $M_{j} \in \mathbb{R}^{+}$is such that $e_{j}(T x, T z) \leq M_{j} \rho_{j}(x-z)$ for all $x, z \in X$ then $\phi_{2 j}$ is $\rho_{j}$-continuous hence $\tau$-continuous.

Proof Let $x \in X$ and $\left\{x_{\lambda}\right\}$ be a net such that $x_{\lambda} \stackrel{\rho_{j}}{\rightarrow} x$. For $z \in T x_{\lambda}$ we have

$$
\begin{aligned}
\phi_{2 j}(x) & =\rho_{j}(x-T x) \leq \rho_{j}\left(x-x_{\lambda}\right)+\rho_{j}\left(x_{\lambda}-T x\right) \\
& \leq \rho_{j}\left(x-x_{\lambda}\right)+\rho_{j}\left(x_{\lambda}-z\right)+e_{j}\left(T x_{\lambda}, T x\right) \\
& \leq\left(1+M_{j}\right) \rho_{j}\left(x-x_{\lambda}\right)+\rho_{j}\left(x_{\lambda}-z\right) .
\end{aligned}
$$

By taking the infimum with respect to $z \in T x_{\lambda}$ we get

$$
\phi_{2 j}(x) \leq\left(1+M_{j}\right) \rho_{j}\left(x-x_{\lambda}\right)+\rho_{j}\left(x_{\lambda}-T x_{\lambda}\right)=\left(1+M_{j}\right) \rho_{j}\left(x-x_{\lambda}\right)+\phi_{2 j}\left(x_{\lambda}\right) .
$$

Thus

$$
\phi_{2 j}(x)-\phi_{2 j}\left(x_{\lambda}\right) \leq\left(1+M_{j}\right) \rho_{j}\left(x_{\lambda}-x\right) \text {. }
$$

Similarly,

$$
\phi_{2 j}\left(x_{\lambda}\right)-\phi_{2 j}(x) \leq\left(1+M_{j}\right) \rho_{j}\left(x_{\lambda}-x\right)
$$

and $\phi_{2 j}$ is $\rho_{j}$-continuous. 
Definition 2.3 For $j \in J, \phi_{j}: X \rightarrow[0, \infty)$ will be the function defined by $\phi_{j}(x)=\phi_{1 j}(x)+$ $\phi_{2 j}(x)$.

Lemma 2.4 Let $A \subset X$ be any closed subset, and suppose that $\phi_{1 j}$ and $\phi_{2 j}$ are $\rho_{j}$-lsc. Suppose that $T: A \rightarrow 2^{A}$ is such that $T x$ is $\rho_{j}$-sequentially compact and for each $x \in A$ and each $n \in \mathbb{N}$ there exists $y_{n} \in T x$ with the following properties: $\phi_{j}\left(y_{n}\right) \leq \phi_{1 j}(x)+\frac{1}{n}$ and $\rho_{j}\left(x-y_{n}\right)<$ $\phi_{2 j}(x)+\frac{1}{n}$. Then there exists $y \in$ Tx such that $\phi_{j}(y)+\rho_{j}(x-y) \leq \phi_{j}(x)$.

Proof Let $\left\{y_{n}\right\} \subset T x$ be a sequence such that each $y_{n}$ satisfies the conditions as in the statement of Lemma 2.4. Since $T x$ is $\rho_{j}$-sequentially compact, there is a subsequence $\left\{y_{n_{k}}\right\}_{k \in \mathbb{N}}$, $\rho_{j}$-convergent to some, not necessarily unique, $y \in T x$. Then

$$
\phi_{j}\left(y_{n_{k}}\right)+\rho_{j}\left(x-y_{n_{k}}\right) \leq \phi_{1 j}(x)+\frac{1}{n_{k}}+\phi_{2 j}(x)+\frac{1}{n_{k}}=\phi_{j}(x)+\frac{2}{n_{k}} .
$$

By taking liminf over $k$ on both sides of the inequality we have

$$
\phi_{j}(y)+\rho_{j}(x-y) \leq \phi_{j}(x)
$$

as desired.

To close this section we define the sets that we will consider as target values for the dynamical systems in Theorem 3.2. Note that Lemma 2.4 is tailored to provide conditions for these sets to be nonempty.

Definition 2.5 For each $x \in X$ and each index $j \in J$ let

$$
C_{x}^{j}=\left\{y \in T x: \phi_{j}(y)+\rho_{j}(x-y) \leq \phi_{j}(x)\right\} .
$$

Lemma 2.6 If $\phi_{j}$ is lsc then the set $C_{x}^{j}$ is $\rho_{j}$-closed.

Proof Let $\left(y_{n}\right)$ be a sequence in $C_{x}^{j}$ such that $y_{n} \stackrel{\rho_{j}}{\rightarrow} y \in X$.

Note that $\phi_{j}\left(y_{n}\right)+\rho_{j}\left(x-y_{n}\right) \leq \phi_{j}(x)$ implies that

$$
\phi_{j}(y)+\rho_{j}(x-y) \leq \liminf \phi_{j}\left(y_{n}\right)+\lim \rho_{j}\left(x-y_{n}\right) \leq \phi_{j}(x) .
$$

Hence $y \in C_{x}^{j}$.

Corollary 2.7 If $\phi_{j}$ is lsc and for each $y \in C_{x}^{j}$ and $y+Z_{\rho_{j}} \subset C_{x}^{j}$, where $Z_{\rho_{j}}=\left\{x \in X: \rho_{j}(x)=0\right\}$ is the zero set of $\rho_{j}$, then $C_{x}^{j}$ is a $\rho_{j}$-closed linear subspace of $X$.

Definition 2.8 Let $A \subset X$ be any closed subset. If $T: A \rightarrow 2^{A}$ is a dynamical system and $\rho_{j}$ is one of the seminorms defining the topology $\tau, x^{*} \in A$ is a $\rho_{j}$-critical point of $T$ if $T x^{*} \subset x^{*}+Z_{\rho_{j}}$.

Note that when $\rho_{j}$ is a norm, to be a $\rho_{j}$-critical point means that $T x^{*}=\left\{x^{*}\right\}$. 


\section{Modified Pareto case}

Recall that the topology $\tau$ in the lcs $X$ is generated by a saturated family of seminorms $\left\{\rho_{j}: j \in J\right\}$. Theorem 1 of [11] provides with sufficient conditions for a given dynamical system $T: A \rightarrow 2^{A}$, over a locally complete subset $A$ of $X$, to have critical points. In [11], Theorem 1 , one essentially sees that if for every $x \in A$ and every $u \in T x, c_{j} \rho_{j}(x-u) \leq$ $\Phi(x)-\Phi(u)$ for every $j \in J$ and for some function $\Phi: A \rightarrow \mathbb{R}$ lsc and bounded below $\left(c_{j}\right.$ are positive scalars such that $\bigcap_{j \in J}\left\{x \in X: c_{j} \rho_{j}(x) \leq 1\right\}$ is a nonzero Banach disk) then $T$ has a critical point. In our setting, if we suppose that, for a fixed $j \in J, A$ is $\rho_{j}$-complete and $\Phi: A \rightarrow \mathbb{R}$ is $\rho_{j}$-lsc, that is, $\rho_{j}\left(x_{n}-x\right) \rightarrow 0$ implies $\Phi(x) \leq \liminf _{n} \Phi\left(x_{n}\right)$ (which in turn implies $\tau$-lsc of $\Phi)$ then we have the following proposition.

Proposition 3.1 If for each $x \in A$ and each $u \in T x$ we have $\rho_{j}(x-u) \leq \Phi(x)-\Phi(u)$, then $T$ has a $\rho_{j}$-critical point, $x_{j}^{*} \in A$.

The proof goes along the same lines as that of Theorem 1 in [11] and we omit it.

Theorem 3.2 (Main Result) Let $T: A \rightarrow 2^{A}$ be a dynamical system and for each $j \in J$ let $T_{j}: A \rightarrow 2^{A}$ be the dynamical system defined by $T_{j}(x)=C_{x}^{j}$. Suppose that the following conditions are satisfied:

1. The seminorm $\rho_{j}$, from the family of seminorms defining the topology $\tau$, is such that the subset $A$ is $\rho_{j}$-sequentially complete.

2. The function $\phi_{j}$ (see definition 2.3) satisfies the condition $\phi_{j}(x) \leq \liminf \phi_{j}\left(x_{n}\right)$ whenever $\rho_{j}\left(x-x_{n}\right) \rightarrow 0$.

3. $C_{x}^{j} \neq \emptyset$ for all $x \in A$.

Then there exists $x_{j}^{*} \in A$ such that $T_{j} x_{j}^{*} \subset x_{j}^{*}+Z_{\rho_{j}}$, that is, $x_{j}^{*}$ is a $\rho_{j}$-critical point of the function $T_{j}$.

Proof The hypotheses of this theorem are essentially the same as those of Proposition 3.1, with $T=T_{j}$. The fact that the function $\phi_{j}$ is $\rho_{j}$-lsc implies that the set $T_{j} x=C_{x}^{j}$ is $\rho_{j}$-closed for each vector $x \in A$. Then, for all $y \in T_{j} x=C_{x}^{j}$,

$$
y+Z_{\rho_{j}}=\overline{\{y\}}^{\rho_{j}} \subset T_{j} x .
$$

On the other hand, the fact that $A$ is $\rho_{j}$-sequentially complete implies that the set $T_{j} x=$ $C_{x}^{j}$ is also $\rho_{j}$-sequentially complete. Hence, by Proposition 3.1, we see that there exists $x_{j}^{*} \in A$ such that

$$
T_{j} x_{j}^{*} \subset x_{j}^{*}+Z_{\rho_{j}}
$$

as desired.

Corollary 3.3 If $x_{j}^{*} \in T_{j} x_{j}^{*}$ then $T_{j} x_{j}^{*}=x_{j}^{*}+Z_{\rho_{j}}$.

A weak version (in the sense of weak topology) of Theorem 3.2 can be obtained as follows. Consider $X^{\prime}=(X, \tau)^{\prime}$, the topological dual of $X$, and the saturated family of seminorms $\left\{\rho_{f}: f \in X^{\prime}\right\}$, where $\rho_{f}(x)=|f(x)|$ and $Z_{f}=\operatorname{ker}(f)$. Assume as before that $A \subset X$ is $\rho_{f}$-complete for some fixed, but arbitrary, $f \in X^{\prime}$, and let $\Phi: A \rightarrow \mathbb{R}$ be a lsc function. Then we have the following. 
Proposition 3.4 If each $x \in A$ and each $u \in T x$ satisfies $|f(x-u)| \leq \Phi(x)-\Phi(u)$, then the dynamical system $T$ has a $\rho_{f}$-critical point $x_{f}^{*} \in A$, that is,

$$
T x_{f}^{*} \subset x_{f}^{*}+Z_{\rho_{f}}=x_{f}^{*}+Z_{f} .
$$

We also have the following restatement of Theorem 3.2, where the continuous linear functions $f \in X^{\prime}$ take the place of the indices $j \in J$.

Theorem 3.5 For each $f \in X^{\prime}$, let $T_{f}^{\prime}: A \rightarrow 2^{A}$ denote the function given by $T_{f}^{\prime}(x)=C_{x}^{f}$. Suppose there exists a seminorm, $\rho_{f}(x)=|f(x)|$ with $f \in X^{\prime}$, such that:

1. The function $\phi_{f}=|f|$ satifies $\phi_{f}(x) \leq \liminf \phi_{f}\left(x_{n}\right)=\liminf _{n}\left|f\left(x_{n}\right)\right|$ (lower semicontinuity).

2. $C_{x}^{f} \neq \emptyset$ for all $x \in A$.

Then there exists $x_{f}^{*} \in A$ such that $T_{f}^{\prime} x_{j}^{*} \subset x_{f}^{*}+Z_{f}$.

Proof Just notice that if we consider the weak topology in place of $\tau$ on $X$, then we have the same hypotheses as in Theorem 3.2 above.

\section{Applications to fixed point theory}

With the tools we have developed so far we can present a couple of interesting applications to fixed point theory. Let $(X, \tau)$ be a complete locally convex topological vector space and take $f \in X^{\prime}, A \subset X$ a $\tau$-closed subset, and $0<M<1$. Let $h: A \rightarrow A$ be any function such that

$$
|f(h(x))-f(h(z))| \leq M|f(x)-f(z)| \quad \text { for all } x, z \in A .
$$

Theorem 4.1 Under the above assumptions there exists $x^{*} \in A$ such that, for all $k \in \mathbb{N}$,

$$
f\left(x^{*}\right)=f\left(h\left(x^{*}\right)\right)=f\left(h^{k}\left(x^{*}\right)\right) .
$$

Equivalently, for all $k \in \mathbb{N}, \rho_{f}\left(x^{*}-h^{k}\left(x^{*}\right)\right)=0$. Moreover, if $x^{* *} \in A$ also satisfies (5) then $f\left(x^{*}\right)=f\left(x^{* *}\right)$.

First note that Theorem 4.1 fails for $M=1$. Indeed, in [22] Khamsi provides an example [22], Example 1, to answer Kirk's problem (in the negative) on the existence of fixed points for a map $T: X \rightarrow X$ such that, for all $x \in X$ and for some positive function $\eta, \eta(d(x, T x)) \leq$ $\phi(x)-T(\phi(x))$ ( $\phi$ is a non-negative lsc function). Here we see that for $M=1$ and $A=$ $\left\{x_{1}, x_{2}, \ldots\right\} \subset X=\mathbb{R}$ with $x_{n}=1+\frac{1}{2}+\cdots+\frac{1}{n}$; if we take $f=I \in(\mathbb{R})^{\prime}$ we have the inequality

$$
\left|h\left(x_{n}\right)-h\left(x_{m}\right)\right| \leq\left|x_{n}-x_{m}\right| \quad \text { for all } n, m \in \mathbb{N}
$$

and then the function $h\left(x_{n}\right)=x_{n+1}$ does not have a fixed point. Note that the set $A$ is closed but it is not bounded.

Also note that if $A=X$ then as a consequence of inequality (4) we see that $\hat{h}: X / Z_{f} \rightarrow$ $X / Z_{f}$, such that $\hat{h}([x])=[h(x)]$ is a function. Furthermore, if $A=X$ and the identities in (5) hold, then the function $\hat{h}$ has a unique fixed point. 
Proof of Theorem 4.1. Let $T: A \rightarrow 2^{A}$ defined by $T x=\left\{x, h(x), h^{2}(x), \ldots\right\}$. Then we have

$$
|f(h(x)-h(z))|=|f(h(x))-f(h(z))| \leq M|f(x)-f(z)|=M|f(x-z)|
$$

for every $x, z \in A$.

Consider $e_{f}:\{T x: x \in X\} \times\{T z: z \in X\} \rightarrow[0, \infty)$ given by

$$
e_{f}(T x, T z)=\sup \{|f(y-T z)|: y \in T z\}=\sup \left\{\rho_{f}(y-T z): y \in T x\right\}
$$

Observe that since $T x \supset T h(x) \supset T h^{2}(x) \supset \cdots$ we have $e_{f}\left(T h^{k+m}(x), T h^{k}(x)\right)=0$ for all $k, m \in \mathbb{N}$. Now $T x$ is not necessarily closed or bounded, however, if $k \leq m$,

$$
\left|f\left(h^{k}(x)\right)-f\left(h^{m}(z)\right)\right| \leq M^{k}\left|f(x)-f\left(h^{m-k}(z)\right)\right|
$$

Thus,

$$
\begin{aligned}
\inf _{m \geq k}\left\{\left|f\left(h^{k} x\right)-f\left(h^{m} z\right)\right|\right\} & \leq \inf _{m \geq k}\left\{M^{k}\left|f(x)-f\left(h^{m-k} z\right)\right|\right\} \\
& \leq M^{k}|f(x)-f(z)| \\
& =M^{k}|f(x-z)| .
\end{aligned}
$$

As a consequence we see that the function $e_{f}$ satisfies $e_{f}(T x, T z) \leq M|f(x-z)|$ for all $x, z \in A$.

Note that for the given function $h$ and the seminorm $\rho_{f}$ we can use Proposition 2.1 to see that the function $\phi_{1 f}(x)=\operatorname{diam}_{f} T x$ is Lipschitz and thus $\rho_{f}$-uniformly continuous. Also, since $x \in T x, \phi_{2 f}(x)=0$ and $\phi_{f}(x)=\phi_{1 f}(x)$. Therefore $\phi_{f}$ is $\rho_{f}$ uniformly continuous and satisfies condition (2) in Theorem 3.2. Observe that since $x \in T x$ we have trivially from Lemma 2.4 that $\phi_{f}(x)+\rho_{f}(x-x)=\phi_{f}(x)$. Also $x \in C_{x}^{f}=\left\{y \in T x \mid \phi_{f}(y)+\rho_{f}(x-y) \leq \phi_{f}(x)\right\}$, that is, $C_{x}^{f} \neq \emptyset$, which is condition (3) in Theorem 3.2.

By Lemma 2.6, and since $\rho_{f}$ is continuous, we see that $C_{x}^{f}$ is $\rho_{f}$-closed, thus $\rho_{f}$ sequentially complete since $X$ is $\rho_{f}$-sequentially complete. By Theorem 3.2 we conclude that, for the map $T_{f}: A \rightarrow 2^{A}$, defined via $T_{f}(x)=C_{x}^{f}$, there exists $x^{*} \in A$ such that $T_{f}\left(x^{*}\right) \subset x^{*}+Z_{f}$.

Now, since $x^{*} \in T_{f}\left(x^{*}\right)$, by Corollary 3.3 we see that $T_{f}\left(x^{*}\right)=x^{*}+Z_{f}$. Hence, for each $y \in$ $T x^{*}$ there exists $z \in Z_{f}(f(z)=0)$ such that $y=x^{*}+z$. From which we obtain $\operatorname{diam}_{f} T x^{*}=0$. That is, $f\left(x^{*}\right)=f\left(h^{k}\left(x^{*}\right)\right)$ for all $k \in \mathbb{N}$. Equivalently $\rho_{f}\left(x^{*}-h^{k}\left(x^{*}\right)\right)=0$ for all $k \in \mathbb{N}$.

As for the uniqueness, if $x^{* *} \in X$ is such that $f\left(x^{* *}\right)=f\left(h^{k}\left(x^{* *}\right)\right)$ for all $k \in \mathbb{N}$, we would have

$$
\left|f\left(x^{*}-x^{* *}\right)\right|=\left|f\left(h\left(x^{*}\right)\right)-f\left(h\left(x^{* *}\right)\right)\right| \leq M\left|f\left(x^{*}\right)-f\left(x^{* *}\right)\right|=M\left|f\left(x^{*}-x^{* *}\right)\right| .
$$

Thus $1 \leq M$, which contradicts our hypothesis. This concludes Theorem 4.1.

There is an interesting connection with the condition of metrical inwardness and the corresponding fixed point theorem in Caristi [23], p.247. Metrical inwardness hold in our setting if we take a fixed function $f \in X^{\prime}$ such that for each $x \in A$ there exists $u \in A$ such that 
$f(x-u)$ and $f(u-h x)$ are both positive numbers (or both negative) where $h$ is a function satisfying the conditions of Theorem 4.1.

All we did in the previous paragraphs can be repeated if we change the weak topology for a generic Hausdorff topology for a lcs $(X, \tau)$. Indeed, if we use a fixed seminorm $\rho_{j}$ among those that generate the topology $\tau$, then we have the following result corresponding to Proposition 4.1.

Proposition 4.2 Let $(X, \tau)$ be a complete locally convex topological vector space, take $\rho_{j}$ a seminorm, from those that generate the topology $\tau$. Let $A \subset X$ a closed subset and let $0<M<1$. Take $h: A \rightarrow A$ defined as a function not necessarily linear or continuous and such that for $\rho_{j}$ we have

$$
\rho_{j}(h(x)-h(z)) \leq M \rho_{j}(x-z) \quad \text { for all } x, z \in A .
$$

Then there exists $x^{*} \in A$ such that

$$
x^{*}-h^{k}\left(x^{*}\right) \in Z_{\rho_{j}} \quad \text { for all } k \in \mathbb{N} \text {. }
$$

Equivalently

$$
\rho_{j}\left(x^{*}-h\left(x^{*}\right)\right)=\rho_{j}\left(x^{*}-h^{k}\left(x^{*}\right)\right)=0 \quad \text { for all } k \in \mathbb{N} .
$$

If $x^{* *} \in A$ also satisfies (7) we have, $\rho_{j}\left(x^{*}-x^{* *}\right)=0$.

A consequence of (6) for $A=X$ is that the relation $\hat{h}: X / Z_{\rho_{j}} \rightarrow X / Z_{\rho_{j}}$ defined by $\hat{h}([x])=$ $[h(x)]$ is a function. A consequence of (7) for $A=X$, is that the function $\hat{h}$ has a unique fixed point.

Proof of Proposition 4.2 To prove Proposition 4.2 let us define the function $T: X \rightarrow 2^{X}$ via $T x=\left\{x, h(x), h^{2}(x), \ldots\right\}$.

Then we have $\rho_{j}(h(x)-h(z)) \leq M \rho_{j}(x-z)$ for all $x, z \in A$. Note that $T x \supset T h(x) \supset$ $T h^{2}(x) \supset \cdots$, which means that $e_{f}\left(T h^{k+m}(x), T h^{k}(x)\right)=0$ for all $k, m \in \mathbb{N}$. Recall that $T x$ is not necessarily closed but it is bounded. Furthermore, if $k \leq m$,

$$
\rho_{j}\left(h^{k}(x)-h^{m}(z)\right) \leq M^{k} \rho_{j}\left(x-h^{m-k}(z)\right),
$$

and then

$$
\inf _{m \geq k}\left\{\rho_{j}\left(h^{k} x-h^{m} z\right)\right\} \leq \inf _{m \geq k}\left\{M^{k} \rho_{j}\left(x-h^{m-k} z\right)\right\} \leq M^{k} \rho_{j}(x-z)
$$

The trick now is to follow the argument in Theorem 4.1 but replacing the seminorm $\rho_{f}$ by the seminorm $\rho_{j}$ in order to get $x^{*} \in X$ such that, by Theorems 2.4, 3.2, and Corollary 3.3, ${\overline{T x^{*}}}^{\rho_{j}}=x^{*}+Z_{\rho_{j}}$. Finally, since $\hat{0} \in Z_{\rho_{j}}$ we obtain $x^{*} \in T x^{*}$. Uniqueness is proved in the same way as in Theorem 4.1.

Note that in Theorem 4.1 if we had $x^{*} \in T x^{*}$ and $x^{* *} \in T x^{* *}$, that is, $x^{*}=h^{k}\left(x^{*}\right)$ and $x^{* *}=h^{j}\left(x^{* *}\right)$, where $k, j \in \mathbb{N}$ are the minimum values that satisfy these equalities, then we 
would have, for each $n \in \mathbb{N}, x^{*}=h^{n k}\left(x^{*}\right)$, and $x^{* *}=h^{n j}\left(x^{* *}\right)$. Thus, for $j \leq k$,

$$
\left|f\left(x^{*}-x^{* *}\right)\right|=\mid f\left(h^{n k}\left(x^{*}\right)-h^{n j}\left(x^{* *}\right)\left|\leq M^{n j}\right| f\left(h^{n k-n j}\left(x^{*}\right)-x^{* *}\right) \mid .\right.
$$

Since $\left\{h^{n(k-j)}\left(x^{*}\right) \mid n \in \mathbb{N}\right\}$ is bounded, since it is finite with at most $k$ points, and $0<M<1$, the sequence $\left\{M^{n j}\left|f\left(h^{n(k-j)}\left(x^{*}\right)-x^{* *}\right)\right|\right\}$ converges to 0 as $n \rightarrow \infty$; in other words, $\mid f\left(x^{*}-\right.$ $\left.x^{* *}\right) \mid=0$ hence $\left|f\left(h\left(\left(x^{*}\right)\right)-h\left(x^{* *}\right)\right)\right|=0$. We conclude that $\rho_{f}\left(x^{*}-x^{* *}\right)=0=\rho_{f}\left(h\left(x^{*}\right)-\right.$ $\left.h\left(x^{* *}\right)\right)$. In general, $\left|f\left(h^{n j}\left(x^{*}\right)-h^{n j}\left(x^{* *}\right)\right)\right|=0=\left|f\left(h^{n k}\left(x^{*}\right)-h^{n k}\left(x^{* *}\right)\right)\right|$.

As another application of our results, we show how the Azé and Corvellec theorem ([9], Theorem 2.3) follows easily from Theorem 3.5. Azé and Corvellec defined the set of fixed points for $T$ as $F_{T}=\{x \in X: x \in T x\}$. For the seminorms $\rho_{j}$ the set of fixed points of $T$ is described as $F_{T}^{j}=\left\{x \in X \mid \rho_{j}(x-T x)=0\right\}$ or in the case of $\rho_{f}$, the set of fixed point for $T$ is $F_{T}^{f}=\{x \in X|| f(x-T x) \mid=0\}$. Note that this sets are $\rho_{j}$-closed (respectively, $\rho_{f}$-closed). In [9], Azé and Corvellec proved that for all $x \in X, \rho_{j}\left(x-F_{T}^{j}\right) \leq \rho_{j}(x-T x)$ $\left(\left|f\left(x-F_{T}^{f}\right)\right| \leq|f(x-T x)|\right)$. We discuss the case for $\rho_{f}$ (the case for $\rho_{j}$ is analogous). Let

$$
A_{f}=\left\{x \in X|| f\left(x-F_{T}^{f}\right)|>| f(x-T x) \mid=\phi_{2 f}(x)\right\} .
$$

We can prove, under the conditions of Propositions 2.1 and 2.2, which we assume, that $A_{f}=\emptyset$. Indeed, define the function $\psi_{f}:(X, d) \rightarrow \mathbb{R}$ by

$$
\psi_{f}(x)=\left|f\left(x-F_{T}^{f}\right)\right|-|f(x-T x)|=\left|f\left(x-F_{T}^{f}\right)\right|-\phi_{2 f}(x) .
$$

Then we have

$$
A_{f}=\left\{x \in X: \psi_{f}(x)>0\right\}=\psi_{f}^{-1}(0, \infty)
$$

Thus if the function $\psi_{f}$ is lsc then $A_{f}$ is an open set, and $A_{f} \cap F_{T}^{f}=\emptyset$. Consider the function $\gamma_{f}(x)=\left|f\left(x-F_{T}^{f}\right)\right|=\inf \left\{|f(x-z)|: z \in F_{T}^{f}\right\}$, which is always continuous. Then, under the conditions of Propositions 2.1 and 2.2, the function $\phi_{2 f}$ is continuous. Thus, we see that the function $\psi_{f}(x)=\gamma_{f}(x)-\phi_{2 f}(x)$ is continuous, giving us that $A_{f}$ is open. If $A_{f} \neq \emptyset$, take $x \in A_{f}$ and $r>0$ such that

$$
D_{r}^{f}(x)=\{z \in X:|f(z-x)| \leq r\} \subset A_{f}
$$

Note that $D_{r}^{f}(x)$ is $\rho_{f}$-closed and $\rho_{f}$-bounded.

If we define $T^{\prime \prime}: D_{r}^{f}(x) \rightarrow 2^{X}$ to be $T^{\prime \prime} x=T_{f}^{\prime} x, T^{\prime \prime}=\left.T_{f}^{\prime}\right|_{D_{r}^{f}(x)}$, then $T_{f}^{\prime}$ satisfies the hypotheses of Theorem 3.5. Thus, there exists $z^{*} \in D_{r}^{f}(x) \subset A_{f}$ such that $z^{*} \in T^{\prime \prime} z^{*}$, in other words, $z^{*} \in A_{f} \cap F_{T}^{f}=\emptyset$ and the set $A_{f}$ must be empty.

As as additional consequence (and easy example) of Proposition 3.1 one can get well known results such as the contraction mapping theorem. Indeed, if $(X, \rho)$ is a normed space and $A$ is a nonempty compact subset of $X$ then any contraction $f: A \rightarrow A$ has a fixed point. This follows from Proposition 3.1 by letting $T: A \rightarrow 2^{A}$ as $T x=\{f(x)\}$ and

$$
\Phi(x)=\sum_{k=0}^{\infty} \rho\left(f^{k}(x)-f^{k+1}(x)\right)=\frac{1}{1-c} \rho(x-f(x))
$$


where $0<c<1$ is the contraction constant for $f$. It is easy to show that $\Phi$ is lsc (in fact continuous) and that $\rho(x-f(x))=\Phi(x)-\Phi(f(x))$. Hence $T$ must have critical points which are, by definition of $T$, fixed points for $f$.

\section{Conclusions}

The paper can be considered as an extension of the Pareto optimization criterion to locally complete locally convex vector spaces [11] with some applications to fixed points. Local completeness is a very weak completeness property. This type of spaces is becoming the convenient setting for several applications. Here in this setting we get a fixed point theorem and as an application we obtain the results of Azé and Corvellec's [9].

\section{Acknowledgements}

The authors were partially supported by the Asociación Mexicana de Cultura, A.C. Research by T. Gilsdorf was done while on the faculty at the ITAM. The authors gratefully acknowledge all the remarks and suggestions made by the anonymous referees.

\section{Competing interests \\ The authors declare that they have no competing interests.}

Authors' contributions

All authors contributed equally and significantly in writing this article. All authors read and approved the final manuscript.

\section{Author details}

'Departamento de Matemáticas, ITAM, Río Hondo 1, Ciudad de México, 01080, México. ²Department of Mathematics, Central Michigan University, Mt Pleasant, MI 48858, USA.

\section{Publisher's Note}

Springer Nature remains neutral with regard to jurisdictional claims in published maps and institutional affiliations.

Received: 9 March 2017 Accepted: 6 July 2017 Published online: 01 August 2017

\section{References}

1. Al-Homidan, S, Ansari, QH, Yao, J-C: Some generalizations of Ekeland-type variational principle with applications to equilibrium problems and fixed point theory. Nonlinear Anal. 69(1), 126-139 (2008)

2. Bianchi, M, Kassay, G, Pini, R: Existence of equilibria via Ekeland's principle. J. Math. Anal. Appl. 305(2), $502-512$ (2005)

3. Bianchi, M, Kassay, G, Pini, R: Ekeland's principle for vector equilibrium problems. Nonlinear Anal. 66(7), 1454-1464 (2007)

4. Bosch, C, Garcia, A, Garcia, CL: An extension of Ekeland's variational principle to locally complete spaces. J. Math. Anal. Appl. 328(1), 106-108 (2007)

5. Hamel, AH: Equivalents to Ekeland's variational principle in uniform spaces. Nonlinear Anal. 62(5), $913-924$ (2005)

6. Hamel, AH: Phelps' lemma, Daneš' drop theorem and Ekeland's principle in locally convex spaces. Proc. Am. Math. Soc. 131(10), 3025-3038 (2003)

7. Qiu, J: A pre-order principle and set-valued Ekeland variational principle. J. Math. Anal. Appl. 419(2), $904-937$ (2014)

8. Qiu, J: An equilibrium version of set-valued Ekeland variational principle and its applications to set-valued vector equilibrium problems. Acta Math. Sin. Engl. Ser. 33(2), 210-234 (2017)

9. Azé, D, Corvellec, JN: A variational method in fixed point results with inwardness conditions. Proc. Am. Math. Soc. $134(12), 3577-3583(2006)$

10. Lim, TC: A fixed point theorem for weakly inward multivalued contractions. J. Math. Anal. Appl. 243, 323-327 (2000)

11. Bosch, C, Garcia, A: Local completeness and Pareto Optimization. Nonlinear Anal. 73(4), 1098-1100 (2010)

12. Isac, G: Sur l'existence de l'optimum de Pareto. Riv. Mat. Univ. Parma (4) 9, 303-325 (1983) (in French)

13. Abdou, AAN, Khamsi, MA: Fixed points of multivalued contraction mappings in modular metric spaces. Fixed Point Theory Appl. 2014, Article ID 249 (2014)

14. Alzorba, S, Günther, C, Popovici, N, Tammer, C: A new algorithm for solving planar multiobjective location problems involving the Manhattan norm. Eur. J. Oper. Res. 258(1), 35-46 (2017)

15. Castellani, M, Giuli, M: Ekeland's principle for cyclically antimonotone equilibrium problems. Nonlinear Anal., Real World Appl. 32, 213-228 (2016)

16. Zhao, F, Yang, L: Hybrid projection methods for equilibrium problems and fixed point problems of infinite family of multivalued asymptotically nonexpansive mappings. J. Nonlinear Funct. Anal. 2016, Article ID 22 (2016)

17. He, F, Qiu, J: Sequentially lower complete spaces and Ekeland's variational principle. Acta Math. Sin. Engl. Ser. 31(8), 1289-1302 (2015)

18. Qiu, J: Set-valued Ekeland variational principles in fuzzy metric spaces. Fuzzy Sets Syst. 245, 43-62 (2014)

19. Salahuddin, J: General set valued vector variational inequality problems. Commun. Optim. Theory 2017, Article ID 13 (2017)

20. Kriegl, A, Michor, PW: The Convenient Setting: Global Analysis. Amer. Math. Soc., Surveys and Monographs, vol. 53 (1973) 
21. Gilsdorf, T, Khavanin, M: Existence and uniqueness for nonlinear integro-differential equations in real locally complete spaces. Sci. Math. Jpn. 76(3), 395-400 (2013)

22. Khamsi, MA: Remarks on Caristi fixed point theorem. Nonlinear Anal. 71(1-2), 227-231 (2009)

23. Caristi, J: Fixed point theorems for mappings satisfying inwardness conditions. Trans. Am. Math. Soc. 215, 241-251 (1976)

Submit your manuscript to a SpringerOpen ${ }^{\odot}$ journal and benefit from:

- Convenient online submission

$\checkmark$ Rigorous peer review

- Open access: articles freely available online

- High visibility within the field

- Retaining the copyright to your article

Submit your next manuscript at $\boldsymbol{~ s p r i n g e r o p e n . c o m ~}$ 